\title{
The X-ray view of M 33 after ROSAT ${ }^{\star}$
}

\author{
F. Haberl and W. Pietsch \\ Max-Planck-Institut für extraterrestrische Physik, Giessenbachstraße, 85748 Garching, Germany \\ Received 28 February 2001 / Accepted 14 April 2001

\begin{abstract}
We present a catalogue of 184 X-ray sources within $50^{\prime}$ of the nucleus of the local group spiral galaxy M 33. The catalogue is derived from an analysis of the complete set of ROSAT archival data pointed in the direction of M 33 and contains X-ray position, existence likelihood, count rates and PSPC spectral hardness ratios. To identify the sources the catalog was correlated with previous X-ray catalogues, optical and radio catalogues. In addition sources were classified according to their X-ray properties. We find seven candidates for supersoft X-ray sources, of which two may be associated with known planetary nebulae in M 33. The majority of X-ray detected supernova remnants is also detected at radio frequencies and seen in optical lines. The low overall X-ray detection rate of optically selected SNRs can probably be attributed to their expansion into interstellar matter of low density.
\end{abstract}

Key words. galaxies: individual: Messier 33 - galaxies: stellar content - stars: variables: general - X-rays: stars catalogs

\section{Introduction}

Besides M 31 and the Magellanic Clouds M 33 belongs to the galaxies in the Local Group best studied in X-rays. As Sc spiral galaxy at a distance of $795 \mathrm{kpc}$ (van den Bergh 1991) with relatively low inclination of $56^{\circ}$ (Zaritsky et al. 1989) M 33 is ideally suited to investigate the X-ray properties of spiral galaxies.

The Einstein Observatory observed M33 in X-rays detecting diffuse emission from hot gas and seventeen unresolved sources (Long et al. 1981; Markert \& Rallis 1983; Trinchieri et al. 1988). The analysis of ROSAT (Trümper 1982) data from the High Resolution Imager (HRI, David et al. 1996) and the Position Sensitive Proportional Counter (PSPC, Pfeffermann et al. 1987) revealed 57 discrete $\mathrm{X}$-ray sources with luminosities larger than $7 \times 10^{35} \mathrm{erg} \mathrm{s}^{-1}$ (Schulman \& Bregman 1995; Long et al. 1996) from which 20 were detected by both instruments. The ROSAT PSPC and HRI observations confirm the detection of diffuse X-ray emission which may trace the spiral arms within 10' of the nucleus (Long et al. 1996; Schulman \& Bregman 1995).

The dominating X-ray source in M 33 with a luminosity of about $10^{39} \mathrm{erg} \mathrm{s}^{-1}$ is the most luminous X-ray source in the Local Group. The nature of this source which coincides with the optical center of M33 remains unclear.

Send offprint requests to: F. Haberl, e-mail: fwh@mpe.mpg.de

* Table 2 is only available in electronic form at the CDS via anonymous ftp to cdsarc.u-strasbg.fr (130.79.128.5) or via

http://cdsweb.u-strasbg.fr/cgi-bin/qcat?J/A+A/373/438
X-ray variability with a possible periodicity of $\sim 106$ days implies that most of the emission arises from a single object, perhaps from a binary system with a black hole primary of $\sim 10 M_{\odot}$ (Dubus et al. 1997). The first analysis of the ROSAT HRI data indicated extended emission between $5^{\prime \prime}$ and 20" around the nucleus (Schulman \& Bregman 1995). However, a re-analysis of the ROSAT HRI data showed that the nucleus is compatible with an X-ray point source (Pietsch \& Haberl 2000). BeppoSAX observations of M 33 are also in agreement with the black hole model (Parmar et al. 2001).

Eclipses with a period of $3.45 \mathrm{~d}$ and possible $0.31 \mathrm{~s}$ pulsations strongly suggest a high mass X-ray binary nature for one of the other bright sources (Dubus et al. 1999). The majority of the sources is however associated with supernova remnants (SNRs) known from optical (Gordon et al. 1998) and radio surveys (Gordon et al. 1999) of M 33.

Schulman \& Bregman (1995) reported on the analysis of 35 ks ROSAT HRI observations from 1992 January and August while Long et al. (1996) performed a deep $50 \mathrm{ks}$ observation spread between 1991 July and 1993 January using the PSPC. Both sets of observations were centered on the nucleus. During its full lifetime ROSAT observed M 33 for a total of $395 \mathrm{ks}$ with the HRI and $61 \mathrm{ks}$ with the PSPC between 1991 July and 1998 January (see Table 1 where $\mathrm{p}$ and $\mathrm{h}$ in the observation ID indicates PSPC and HRI observations, respectively). Some of the HRI pointings were offset from the nucleus but due to the smaller field of view were all covered completely by the PSPC observations. Here we present the results of an analysis of the 
Table 1. ROSAT observations of M 33 .

\begin{tabular}{|c|c|c|c|c|c|}
\hline ID & Exp. & Start & End & \multicolumn{2}{|c|}{$\begin{array}{l}\text { Pointing center } \\
\text { RA Dec (J2000) }\end{array}$} \\
\hline & {$[\mathrm{s}]$} & \multicolumn{2}{|c|}{ [yymmdd] } & {$[\mathrm{h} \quad \mathrm{m} s]$} & {$\left[\begin{array}{lll}0 & 1 & \prime \prime\end{array}\right]$} \\
\hline $600023 p-0$ & 28690 & 910729 & 910730 & 13350 & 303936 \\
\hline $600020 \mathrm{~h}-0$ & 19188 & 920108 & 920112 & 13353 & 303824 \\
\hline $600020 \mathrm{~h}-1$ & 15963 & 920801 & 920803 & 13353 & 303824 \\
\hline $600023 \mathrm{p}-1$ & 4991 & 920810 & 920810 & 13350 & 303936 \\
\hline $600023 \mathrm{p}-2$ & 16264 & 930107 & 930109 & 13350 & 303936 \\
\hline $600407 \mathrm{p}$ & 3702 & 930205 & 930205 & 13236 & 304536 \\
\hline $600410 p$ & 3458 & 930205 & 930206 & $\begin{array}{lll}13319\end{array}$ & 305248 \\
\hline $600389 p$ & 4474 & 930206 & 930207 & 13412 & 305100 \\
\hline $600487 \mathrm{~h}$ & 30830 & 940727 & 940807 & 13322 & 302436 \\
\hline $600488 \mathrm{~h}$ & 24771 & 940727 & 940807 & $\begin{array}{lll}13307\end{array}$ & 303036 \\
\hline $600486 \mathrm{~h}$ & 16543 & 940801 & 940811 & 13326 & 304500 \\
\hline $600489 \mathrm{~h}$ & 20506 & 940806 & 940808 & $\begin{array}{lll}13307\end{array}$ & 303824 \\
\hline $600485 \mathrm{~h}$ & 17279 & 940806 & 940808 & 13338 & $\begin{array}{lll}30 & 5512\end{array}$ \\
\hline $600483 \mathrm{~h}$ & 26122 & 940806 & 940809 & 13436 & 304912 \\
\hline $600484 \mathrm{~h}$ & 18839 & 940808 & 940809 & 13358 & 305248 \\
\hline $400460 \mathrm{~h}$ & 7968 & 940810 & 940811 & 13350 & 303936 \\
\hline $400746 \mathrm{~h}$ & 24817 & 950115 & 950115 & 13350 & 303936 \\
\hline 400460h-1 & 40961 & 950710 & 950716 & 13350 & 303936 \\
\hline $600911 \mathrm{~h}$ & 48695 & 960118 & 960208 & 13350 & 303936 \\
\hline 600911h-1 & 44658 & 960717 & 960727 & 13350 & 303936 \\
\hline 400903h & 33908 & 970110 & 970114 & 13350 & 303936 \\
\hline $400929 \mathrm{~h}$ & 4225 & 980108 & 980108 & 13350 & 303936 \\
\hline
\end{tabular}

complete set of ROSAT observations with the aim to derive a catalogue of X-ray sources in the direction of M 33.

\section{Data analysis and results}

\subsection{Catalogue of point sources}

To derive a catalogue of discrete X-ray sources in the region of M 33 we followed the guide lines described by Haberl \& Pietsch (1999, hereafter HP99) and Sasaki et al. (2000) who produced PSPC and HRI catalogues for the Large Magellanic Cloud (LMC). The source detection procedures based on combined sliding window and maximum likelihood methods of EXSAS (Zimmermann et al. 1994) were applied to all 22 observations individually and to merged observations to gain sensitivity. To avoid a degradation of the spatial resolution in the images we merged PSPC data only when the pointing direction of the observations was within $2^{\prime}$, resulting in $50.0 \mathrm{ks}$ merged PSPC data centered on the nucleus (corresponding to the data analyzed by Long et al. 1996). Similarly the nine HRI observations centered on the nucleus were combined yielding $237.1 \mathrm{ks}$ of data. Unlike for the investigation of diffuse emission in the nuclear area of M 33 (see below) the data was not screened for high background in the source detection analysis. Further four HRI pointings north-east and three HRI pointings south-west were successively merged and their overlapping regions investigated. The images were co-aligned using the X-ray nucleus (assumed at $\mathrm{RA}=01^{\mathrm{h}} 33^{\mathrm{m}} 50^{\mathrm{s}} .9$ and $\mathrm{Dec}=30^{\circ} 39^{\prime} 36^{\prime \prime}$, J2000.0) and other bright sources as reference. Utilizing the moderate spectral resolution of the PSPC, source detection was run in five energy bands (soft: $0.1-0.4 \mathrm{keV}$, hard: $0.5-2.0 \mathrm{keV}$, hard1: $0.5-0.9 \mathrm{keV}$, hard2: $0.9-2.0 \mathrm{keV}$ and broad 0.1$2.4 \mathrm{keV}$, see also HP99) while for the HRI the full energy band (0.1-2.4 keV) was used. Preliminary results were published in Haberl \& Pietsch (2000).
From the full list of X-ray detections a catalogue of 184 discrete sources within $50^{\prime}$ of the nucleus of M 33 was produced by selecting the detection with the smallest uncertainty in X-ray position. For the sources detected by both instruments the HRI gives generally the smaller positional error (with the exception of only one source). Table 2 lists the source catalogue including $\mathrm{X}$ ray position (Cols. 2 and 3) with $90 \%$ uncertainty radius (Col. 4), likelihood of existence from detection with best position (Col. 5), vignetting corrected exposure times for HRI and PSPC observations (Cols. 6-7), HRI and PSPC count rates (again from observations with best position, Cols. 8-9), PSPC hardness ratios (Cols. 10-11) and remarks on the possible nature of the source (Col. 12). The positional error includes a $2^{\prime \prime}$ systematic error to allow for residual alignment errors. Hardness ratios are defined as $H R 1=(H-S) /(S+H)$ and $H R 2=(H 2-H 1) /(H 1+H 2)$ where $S, H, H 1$ and $H 2$ denote count rates in the $0.1-$ $0.4 \mathrm{keV}, 0.5-2.0 \mathrm{keV}, 0.5-0.9 \mathrm{keV}$ and $0.9-2.0 \mathrm{keV}$ bands, respectively.

\subsection{X-ray images}

To produce clean X-ray images which show besides the point sources also the diffuse emission in the central area of M 33 we merged PSPC and HRI data after careful screening for low-background intervals. A more quantitative analysis of the diffuse emission in M 33 was carried out by Schulman \& Bregman (1995) and Long et al. (1996) and will not be repeated here.

After screening, PSPC data with an exposure of $46.5 \mathrm{ks}$ remained. Exposure corrected images with the nucleus in the center for the broad, soft, hard1 and hard2 energy bands are shown in Fig. 1. The sources from Table 2 are marked on the images. The images detail the data discussed by Long et al. (1996) and clearly show the diffuse emission in particular in the soft, hard1 and broad bands. Point sources and diffuse emission are well separated in the images due to the smoothing with the point spread function (PSF) appropriate for the individual energy bands (see e.g. Pietsch et al. 2000). The diffuse emission follows the inner spiral arms, appears to be highly filamentary, and is more concentrated to the south of the nucleus, an area which is also brighter in the optical and $\mathrm{H} \alpha$ (e.g. Hodge et al. 1999), indicative of enhanced star formation.

To further visualize the filamentary structure of the diffuse emission we merged the ROSAT HRI data centered on the nucleus (pointing direction within $2^{\prime}$ ). In the merging process we corrected for slight pointing shifts between and within the individual observations using the nuclear source as reference. This resulted in $237 \mathrm{ks}$ merged HRI data centered on the nucleus. Screening out times of high background reduced the useful time to $160 \mathrm{ks}$. To be most sensitive for diffuse emission the HRI image was smoothed using a method of adaptive filtering. In this method the lower photon levels in the image are smoothed with wider Gaussian functions than the high intensity levels of point 

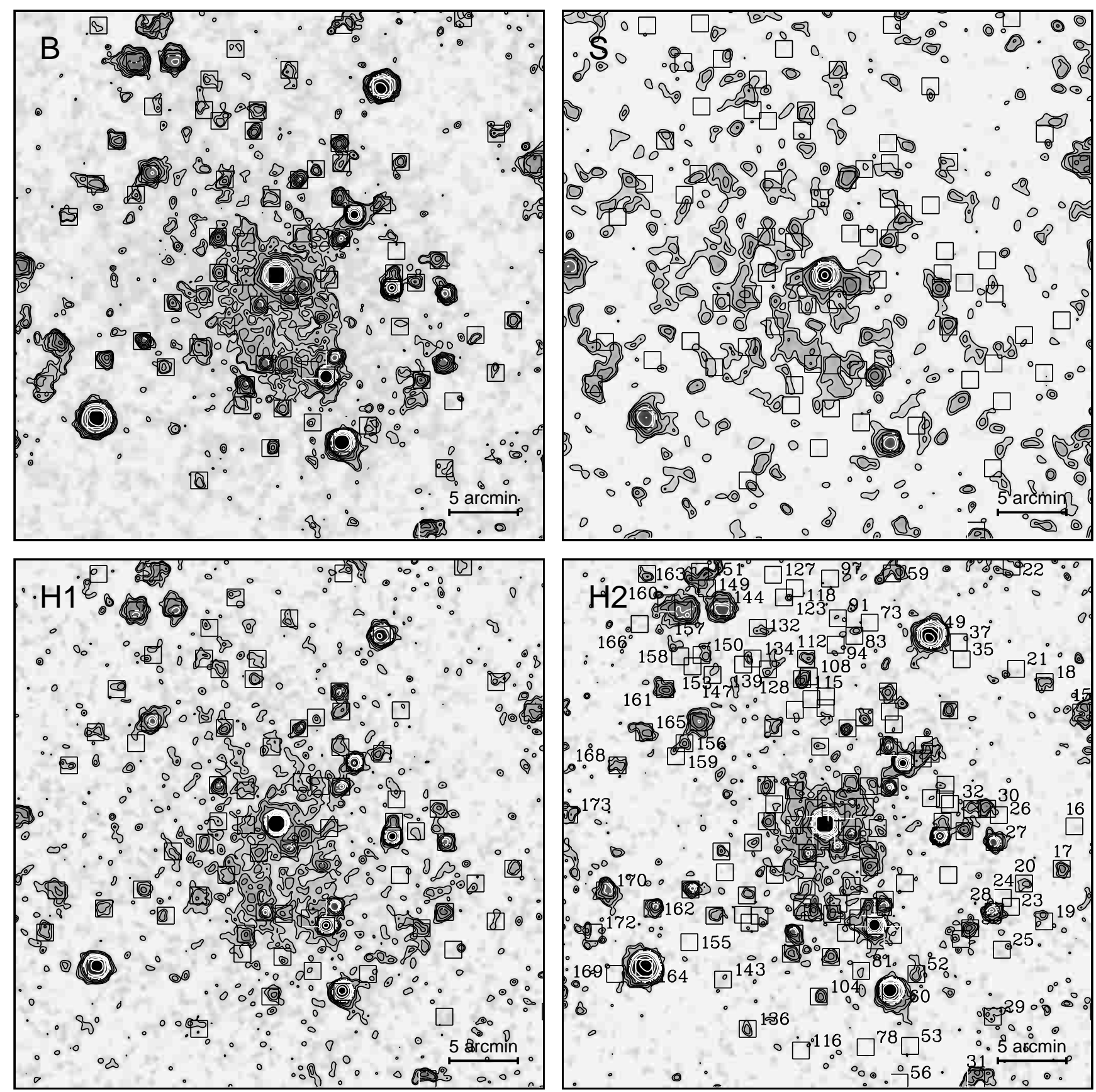

Fig. 1. Smoothed grey-scale ROSAT images of M 33 in four different energy bands (B: 0.1-2.4 keV upper left, S: $0.1-0.4 \mathrm{keV}$ upper right, H1: $0.5-0.9 \mathrm{keV}$ lower left and H2: $0.9-2.0 \mathrm{keV}$ lower right) produced from $46.5 \mathrm{ks}$ of PSPC low-background data centered on the galaxy nucleus. Superimposed contours in the $B$ and $S$ bands are given in units of $\sigma((200$ and 100$) \times$ $10^{-6} \mathrm{cts} \mathrm{s}^{-1}$ arcmin $^{-2}$, respectively) above the background $\left((900\right.$ and 500$) \times 10^{-6} \mathrm{cts} \mathrm{s}^{-1} \operatorname{arcmin}^{-2}$, respectively $)$. In the hard bands $H 1$ and $H 2$ the background is negligible and the contours are in units of 1 photon accumulated within the $F W H M\left(\sim 28^{\prime \prime}\right)$ of the point spread function. One unit is $175 \times 10^{-6} \mathrm{cts} \mathrm{s}^{-1} \operatorname{arcmin}^{-2}$ for the hard bands. Contour levels are 3, 5, 8, 12, 17, 30, 60 and 120 units for all contour plots. The detected discrete X-ray sources are marked with squares and labeled with the catalog number in the $H 2$ image. Numbers for sources in the inner part of the image can be found in Fig. 2.

sources (Ehle et al. 1995). The corresponding image is shown in Fig. 2 again with sources from Table 2 marked, and as contour overlay on a digitized sky survey image in Fig. 3. The comparison with the PSPC images clearly demonstrates the higher spatial resolution of the HRI. The emission is at least partly further resolved into narrow filaments (see e.g. NW and SE of nucleus). However, diffuse emission is clearly remaining besides point sources and these filaments. 

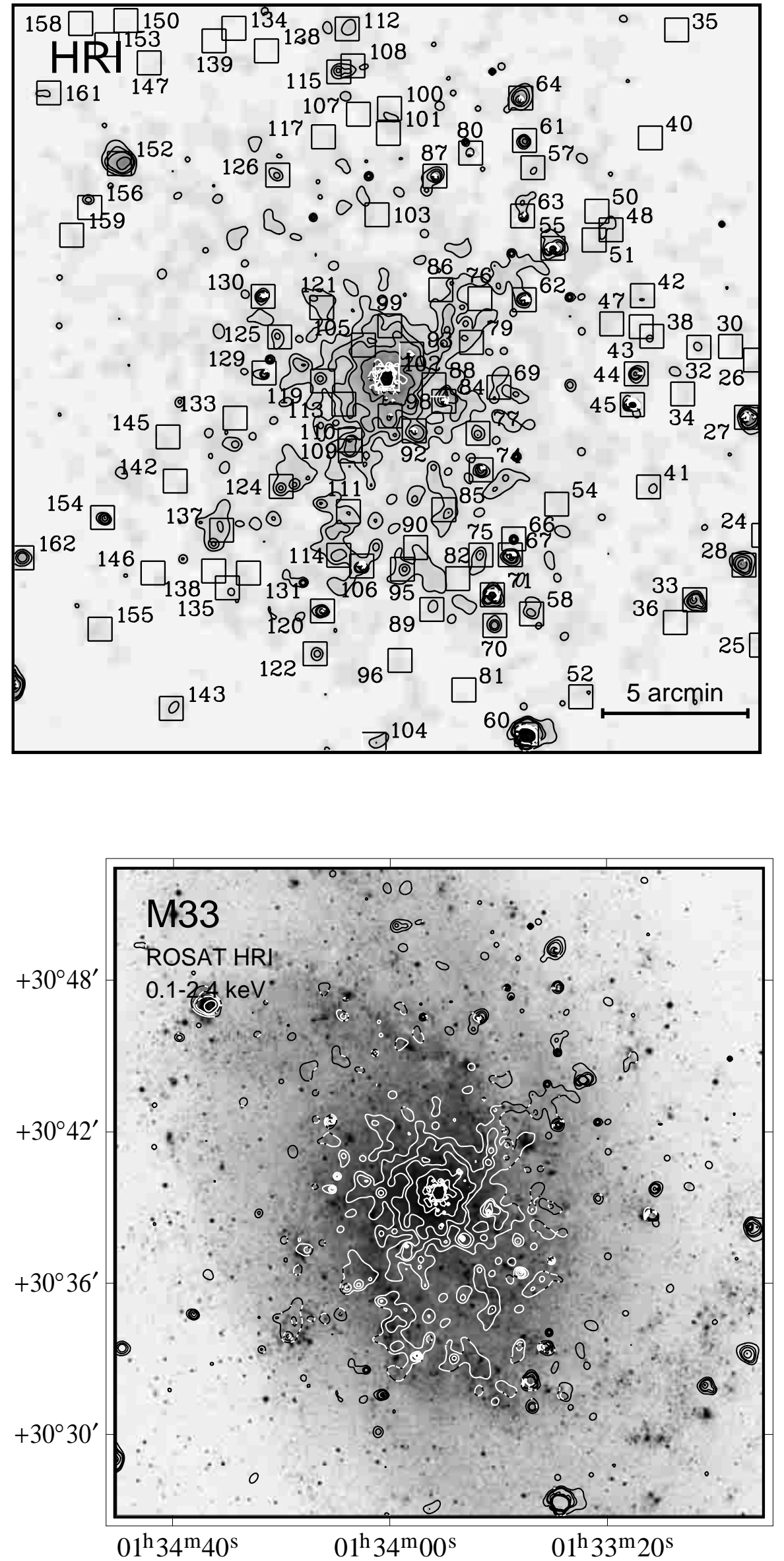

Fig. 2. Smoothed grey-scale ROSAT HRI image of the central region of M 33 utilizing $160 \mathrm{ks}$ of low-background HRI data. Contours are given in units of $\sigma\left(180 \times 10^{-6} \mathrm{cts} \mathrm{s}^{-1} \operatorname{arcmin}^{-2}\right)$ above the background $(2070 \times$ $\left.10^{-6} \mathrm{cts} \mathrm{s}^{-1} \operatorname{arcmin}^{-2}\right)$. Contour levels are $5,7,15,30,60$ and 120 units. Numbered squares mark the catalogued X-ray sources as in Fig. 1.
Fig. 3. Contour plot of the HRI image shown in Fig. 2 overlayed on an optical image of M33 extracted from the digitized sky survey (DSS2). 

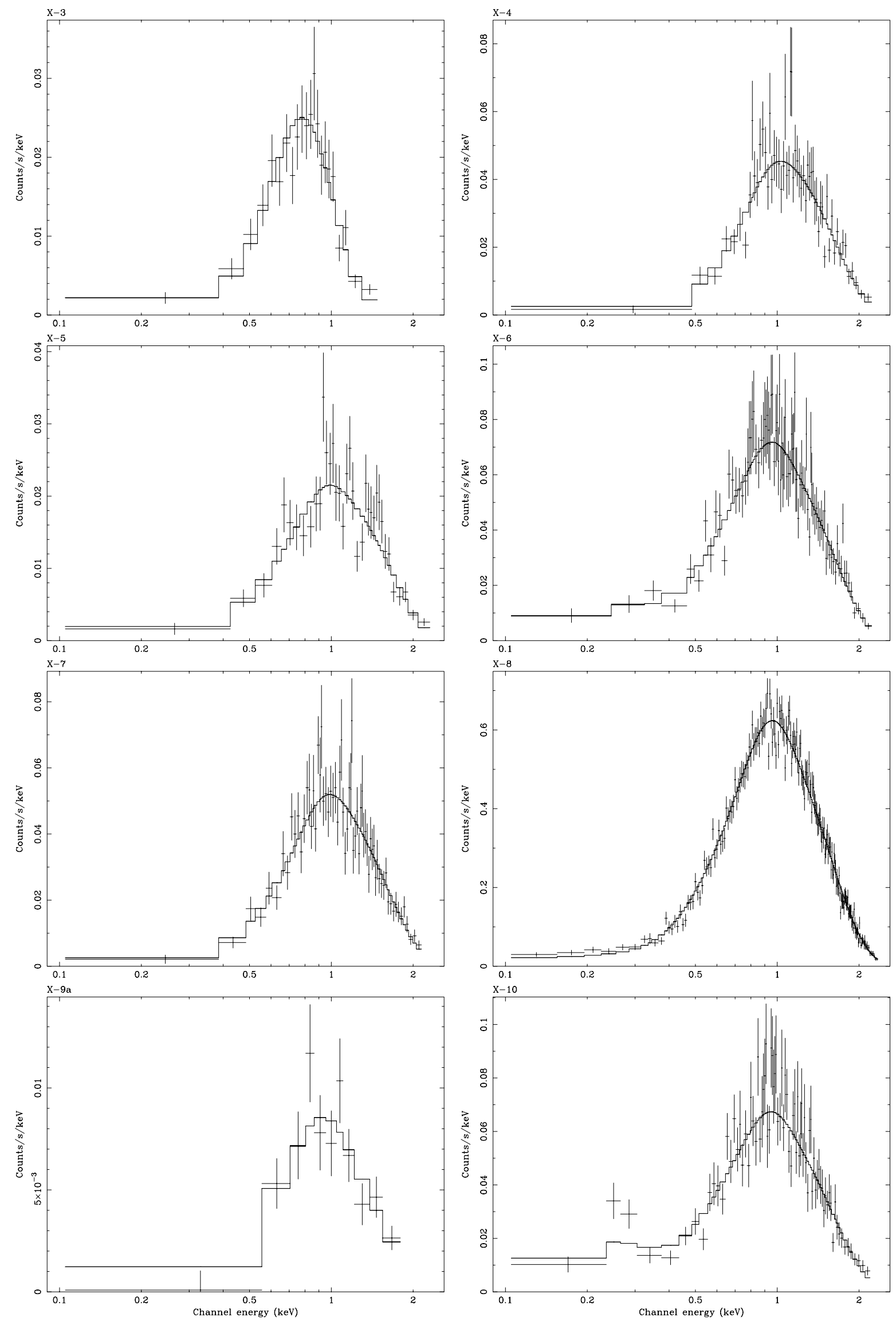

Fig. 4. PSPC spectra of the eight brightest X-ray sources within $20^{\prime}$ of the nucleus of M 33 . The histograms show the best fit power-law model, except for X-3 where a thermal model yields a better fit. 
Table 3. Spectral fit results.

\begin{tabular}{|c|c|c|c|c|c|c|c|c|}
\hline \multirow[t]{2}{*}{ Source } & \multicolumn{3}{|c|}{ Power-law } & \multicolumn{3}{|c|}{ Thermal } & \multirow{2}{*}{ 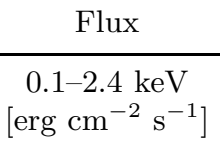 } & \multirow[t]{2}{*}{ Comment } \\
\hline & $\gamma$ & $\begin{array}{c}N_{\mathrm{H}} \\
{\left[10^{21} \mathrm{~cm}^{-2}\right]}\end{array}$ & $\chi_{\mathrm{r}}^{2} / \mathrm{dof}$ & $\begin{array}{c}k T \\
{[\mathrm{keV}]}\end{array}$ & $\begin{array}{c}N_{\mathrm{H}} \\
{\left[10^{21} \mathrm{~cm}^{-2}\right]}\end{array}$ & $\chi_{\mathrm{r}}^{2} / \mathrm{dof}$ & & \\
\hline $45(\mathrm{X}-3)$ & 10 & $9.2 \pm 1.8$ & $1.5 / 19$ & $0.32_{-0.17}^{+0.05}$ & $0.77_{-0.21}^{+4.9}$ & $0.99 / 19$ & $1.4 \times 10^{-13}$ & thermal \\
\hline $49(\mathrm{X}-4)$ & $2.14 \pm 0.68$ & $3.2 \pm 1.4$ & $1.3 / 54$ & $0.56 \pm 0.07$ & $12.0 \pm 0.8$ & $2.1 / 54$ & $6.3 \times 10^{-13}$ & non-thermal \\
\hline $55(\mathrm{X}-5)$ & $1.22_{-0.32}^{+0.48}$ & $0.93_{-0.35}^{+0.8}$ & $1.5 / 34$ & $0.56 \pm 0.09$ & $11.9 \pm 1.0$ & $3.7 / 34$ & $3.4 \times 10^{-13}$ & non-thermal \\
\hline $60(\mathrm{X}-6)$ & $1.48 \pm 0.16$ & $0.73 \pm 0.16$ & $0.94 / 85$ & $>5.1$ & $0.63 \pm 0.09$ & $0.95 / 85$ & $1.1 \times 10^{-12}$ & non-thermal \\
\hline $71(\mathrm{X}-7)$ & $1.86 \pm 0.52$ & $1.9 \pm 0.9$ & $0.95 / 63$ & $0.56 \pm 0.05$ & $11.0 \pm 0.7$ & $2.8 / 63$ & $7.2 \times 10^{-13}$ & non-thermal \\
\hline $102(\mathrm{X}-8)$ & $1.95 \pm 0.13$ & $1.6 \pm 0.2$ & $1.2 / 189$ & $3.7 \pm 0.35$ & $0.96 \pm 0.04$ & $1.3 / 189$ & $8.4 \times 10^{-12}$ & non-thermal \\
\hline $157(\mathrm{X}-9 \mathrm{a})$ & $2.7_{-1.2}^{+2.7}$ & $2.2_{-1.3}^{+4.7}$ & $0.99 / 8$ & $>1.7$ & $0.77_{-0.21}^{+0.62}$ & $1.4 / 8$ & $1.0 \times 10^{-13}$ & non-thermal \\
\hline $164(\mathrm{X}-10)$ & $1.46 \pm 0.15$ & $0.59 \pm 0.11$ & $1.2 / 78$ & $>3.7$ & $0.53 \pm 0.06$ & $1.2 / 78$ & $1.0 \times 10^{-12}$ & non-thermal \\
\hline
\end{tabular}

\subsection{PSPC spectra of bright sources}

For the eight brightest X-ray sources within $20^{\prime}$ of the M 33 nucleus ROSAT PSPC spectra were accumulated. All of these sources were detected in the Einstein data (Trinchieri et al. 1988). We fitted two types of models to the spectra: a power-law and a thermal model (using the collisional equillibrium plasma model MEKAL in XSPEC). The best fit parameters together with the observed flux derived from the better fitting model are summarized in Table 3. X-3 shows the softest spectrum which is consistent with thermal emission and confirms its identification with an SNR detected at optical and radio wavelengths. With a $0.1-2.4 \mathrm{keV}$ luminosity of $1.06 \times 10^{37} \mathrm{erg} \mathrm{s}^{-1}$ it is the X-ray brigthest SNR in M 33 and similar to the brightest SNRs in the LMC and SMC. The spectra of the other seven bright sources are harder, as is shown in Fig. 4 and quantified by the hardness ratios given in Table 2. BeppoSAX spectroscopy of X-4, X-5, $\mathrm{X}-7$ and $\mathrm{X}-10$ shows that the spectra are still consistent with a power-law shape in the $2-10 \mathrm{keV}$ band, while the spectrum of X-6 appears to be significantly more complex (Parmar et al. 2001).

\section{Source identification and classification}

To identify the X-ray sources of M 33 we cross-correlated the catalogue with various other catalogues and data bases like SIMBAD and NED. This includes X-ray catalogues from previous work on Einstein and ROSAT data as well as radio catalogues and the SNR catalogues of Gordon et al. $(1998,1999)$. The identifications based on positional coincidence are indicated in the remarks of Table 2. With increasing source density in the inner parts of M 33 it is however impossible to identify the nature of the X-ray sources from positional coincidence only.

In our work on the LMC we developed a scheme to classify the sources by using their X-ray properties (HP99). In particular we used source extent and spectral characteristics (hardness ratios) to constrain the nature of the X-ray sources. In the case of the nearby LMC SNRs could be resolved by the ROSAT instruments while this is not possible for M 33. For M 33 we used hardness ratios ob- tained from the PSPC count rates for a classification of the $\mathrm{X}$-ray sources and in some cases where count rate statistics allowed time variability studies (on time scales of years between observations) were performed. Figure 5 shows $H R 2$ versus $H R 1$ for the sources detected by the PSPC. As in the case of the LMC (Fig. 4 of HP99) certain regions in the parameter space are populated by only a single type of X-ray source. Because the galactic foreground absorption is similar in the direction of the LMC and M 33 the hardness ratio $H R 1$ (which is most sensitive to absorption) is also similar for objects with the same intrinsic spectrum in both galaxies. Therefore the same borders for dividing regions of different source types could be used. Table 4 summarizes the number of identified and classified X-ray sources. The identifications are based only on positional coincidence and may be accidential in some cases (see discussion below).

\subsection{Supersoft $X$-ray sources}

In addition to previously identified SNRs and X-ray binaries we find seven candidates for supersoft X-ray sources (SSSs). In Fig. 6 HR2 (when available) is plotted as function of distance to the galaxy nucleus. All SSS candidates are found within $10^{\prime}$ of the nucleus. Two of them might be associated with planetary nebulae $(\mathrm{PN})$ from the list of Magrini et al. (2000) supporting their suggested nature. The relatively high $H R 1$ values indicate additional absorption either intrinsic to M 33 or to the source similar as found for the SSS CAL 87 in the LMC (Kahabka et al. 1994) with $H R 1 \sim 0.8$. A comparison of the count rates of the candidate SSSs in M 33 with those in the LMC, simply by scaling with the distance ratio, shows that the M 33 sources also exhibit blackbody $(k T \sim 40 \mathrm{eV})$ luminosities of the order of $10^{38} \mathrm{erg} \mathrm{s}^{-1}$ consistent with the Eddington luminosity for a $1 M_{\odot}$ compact object (Kahabka et al. 1994).

\subsection{Supernova remnants}

Sixteen ROSAT sources coincide in position with known SNRs (Gordon et al. 1998, 1999). Source 95 is however 
Table 4. Summary of identifications and classifications.

\begin{tabular}{|c|c|c|c|}
\hline Source type & classification criteria & identified & classified \\
\hline SSS & $H R 2<-0.70$ & & 7 \\
\hline fg star & $H R 1<0.25 H R 2>-0.70$ EHR $1<0.25$ EHR $2<0.25$ & 4 & 5 \\
\hline SNR & $H R 1>0.5 H R 2>-0.70 H R 2<-0.10$ EHR $1<0.25$ EHR $2<0.25$ & 14 & 3 \\
\hline AGN & Radio source or galaxy, $H R 1>0.25 H R 2>-0.10$ & & 7 \\
\hline X-ray binary & $\mathrm{X}$-ray variable, distance to nucleus $<15^{\prime} H R 1>0.25 H R 2>-0.10$ & 1 & 3 \\
\hline
\end{tabular}

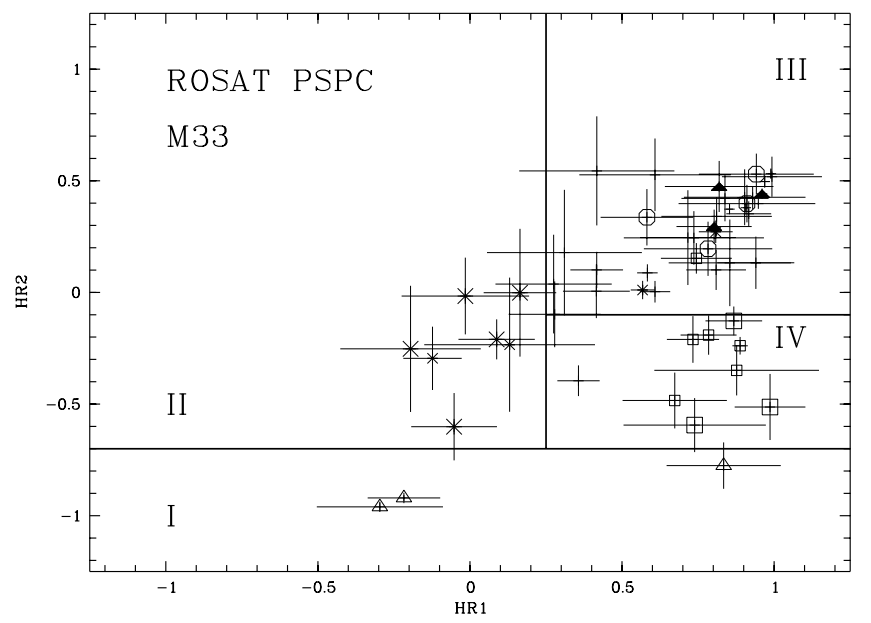

Fig. 5. Hardness ratios of sources detected by the PSPC. Shown are only values with errors smaller than 0.30 on both $H R 1$ and $H R 2$. In part I of the parameter space candidates for SSSs (triangles), in II foreground stars $(x)$ and in IV SNRs (squares) are found. Part III mainly contains X-ray binaries (filled triangles) and AGN (circles), but can also include some foreground stars and SNRs. Small symbols indicate X-ray sources with likely counterpart found from spatial correlations which is supported in most cases by the hardness ratio criteria (only for foreground stars and SNRs). Large symbols mark the candidates classified from hardness ratios only.

more likely a foreground star as it shows the typical hardness ratios for that class of objects. Another two sources show time variability in their X-ray flux; for source 27 with moderate variability the SNR may contribute to the X-ray flux while source 30 was detected in only one observation and the X-ray transient-like behaviour is most likely associated with an X-ray binary. Three X-ray sources which are located in region IV of the hardness diagram (large squares in Fig. 5), where most of the identified SNRs are found, were classified as SNR candidates. The identified SNRs and the new candidates are distributed out to distances of $\sim 15^{\prime}$ from the nucleus.

The majority of twelve SNRs are detected in the radio, optical and X-ray wavelength bands, similar to the SNR census reviewed by Duric (2000). Only two remnants are seen in X-rays and optical but not in the radio band and no SNR was found which is detected in X-rays and radio only. This is in line with the theoretical picture in which radio and X-ray emission depend on the ambient gas density. The low overall number of X-ray detections of optically selected SNRs (98 in Gordon et al. 1998) can probably be

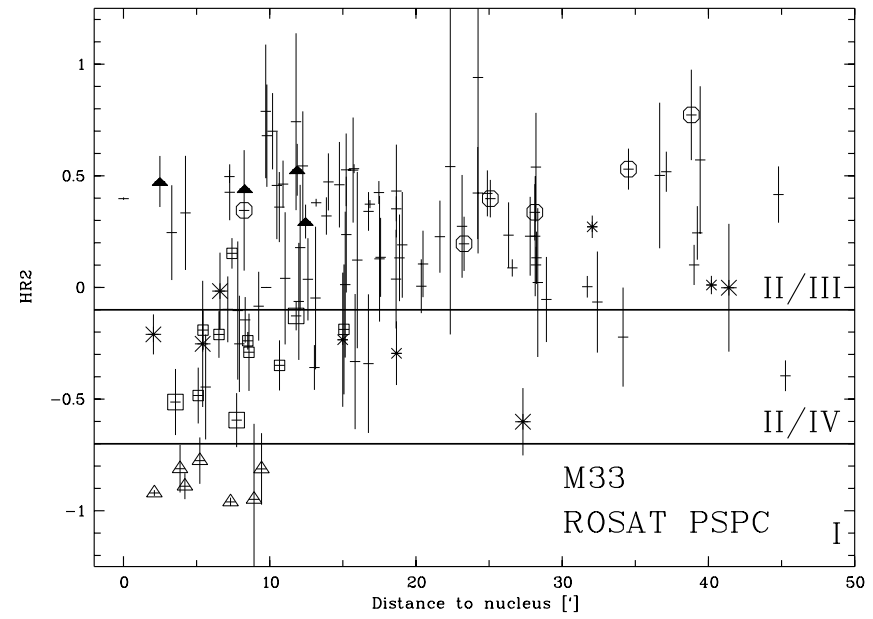

Fig. 6. Hardness ratio $H R 2$ plotted for PSPC-detected sources as a function of their distance to the nucleus of M 33. In the outer parts most sources are probably foreground stars or background AGN (in particular those with $H R 2>0.2$ ).

attributed to their expansion into a low density interstellar medium. The number of eleven X-ray candidates given by Duric (2000) is considerably larger than the three from this work. This is caused by the refined hardness ratio classification used in the current work which allows us to differentiate between supersoft sources, foreground stars and SNRs in the more general class of soft sources used by Duric (2000).

To compare the X-ray luminosity distribution of the SNRs in M 33 with those in the Magellanic Clouds we normalized all PSPC (0.1-2.4 keV) count rates to an assumed LMC distance of $50 \mathrm{kpc}$. For the SMC and M 33 distances of $65 \mathrm{kpc}$ and $795 \mathrm{kpc}$ were used, respectively. The samples of identified SNRs in LMC and SMC were extracted from the PSPC catalogues of HP99 and Haberl et al. (2000), respectively. From our M 33 sample five SNRs are not detected by the PSPC and count rates were converted by multiplying the HRI rate by a factor of 3.0, typical for hard spectra (Sasaki et al. 2000). To convert the PSPC count rate to luminosity we used a non-equillibrium ionization (NEI) model used to fit spectra of SNRs in the LMC (e.g. Hughes et al. 1998). With typical model parameters as temperature $0.6 \mathrm{keV}$, elemental abundance 0.5 solar, ionization time scale $1.0 \times 10^{11} \mathrm{~cm}^{-3} \mathrm{~s}$ and a foreground absorption column density of $6 \times 10^{20} \mathrm{~cm}^{-2}$, this results in a conversion factor of $3.0 \times 10^{36} \mathrm{erg} \mathrm{s}^{-1}$ per 1.0 PSPC $\operatorname{cts~s}^{-1}$. 


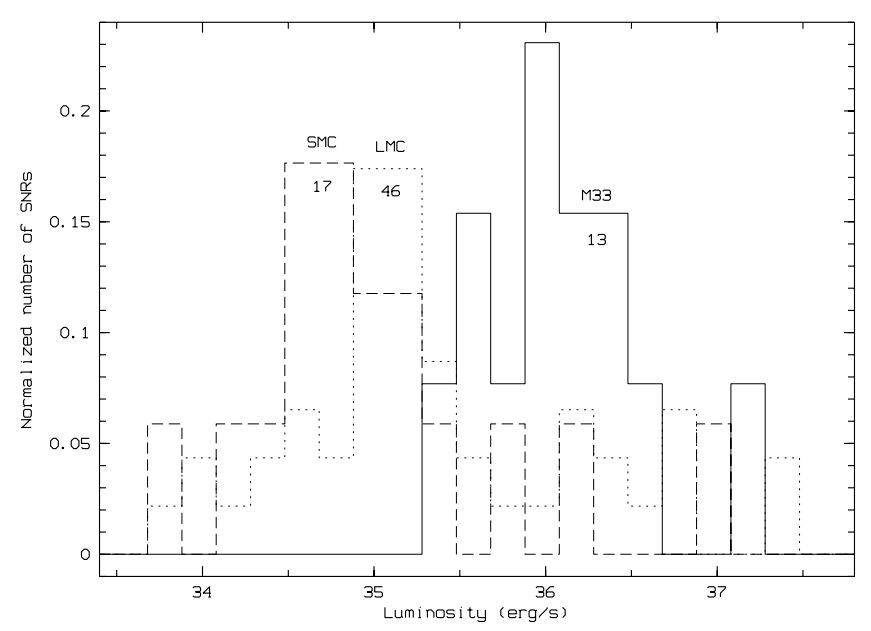

Fig. 7. X-ray luminosity distribution of identified SNRs in M 33 in comparison to LMC and SMC. The number of SNRs is normalized to the total number of SNRs in the galaxy (given below the galaxy name).

The SNR luminosity distributions are compared in Fig. 7. Due to the larger distance of M 33 the SNRs fainter than $1.9 \times 10^{35} \mathrm{erg} \mathrm{s}^{-1}$ are not detected. The ratio of faint to bright (relative to the $1.9 \times 10^{35} \mathrm{erg} \mathrm{s}^{-1}$ ) SNRs in $\operatorname{SMC}(13 / 4=3.25)$ and $\operatorname{LMC}(27 / 19=1.42)$ is quite different with a relatively high number of X-ray bright SNRs in the LMC. This may be caused by the higher metal abundance in the LMC because elements like $\mathrm{O}$, $\mathrm{Ne}, \mathrm{Mg}, \mathrm{Si}$ and Fe produce the major part of the X-ray flux in the ROSAT band. The expected number of faint SNRs in M 33 therefore may vary between 20 and 40, if the conditions resemble more those in the LMC or the SMC, respectively. Monteverde et al. (1997) report an oxygen abundance gradient in M33 investigating the spectra of B-type supergiants. They found the $\mathrm{O}$ abundance $(\mathrm{O} / \mathrm{H})$ decreasing from about solar values near the galaxy nucleus to about 0.34 solar at $15^{\prime}$ off-center. The average value within $15^{\prime}$ is similar to the average metal abundance of 0.5 solar in the LMC (Russell \& Dopita 1992). Therefore, we use the luminosity distribution of SNRs in the LMC to estimate the contribution of faint, still undetected, SNRs in M 33 to the diffuse emission detected in its central region. Integrating the 27 faint LMC SNRs yields a luminosity of $2.3 \times 10^{36} \mathrm{erg} \mathrm{s}^{-1}$ corresponding to $1.7 \times 10^{36} \mathrm{erg} \mathrm{s}^{-1}$ for 20 faint SNRs in M 33. This is far below the luminosity of about $10^{39} \mathrm{erg} \mathrm{s}^{-1}$ found for the diffuse emission in M 33 by Schulman \& Bregman (1995).

\subsection{X-ray binaries}

Apart from the known X-ray binary X-7 (source 71, Dubus et al. 1999) we suggest three X-ray binaries from their time variability (source 110 and the two discussed above). They are also located within $15^{\prime}$ of the nucleus.

To search for additional X-ray binary candidates we correlated our X-ray source catalogue with the list of 51 globular clusters in M 33 from Mochejska et al. (1998).
None of the X-ray sources could be associated with a known globular cluster within the error circle. Supper et al. (1997) found 24 X-ray sources (expected chance coincidences subtracted) correlating with globular clusters in M31. The data base of optically selected globular clusters in M31 used by Supper et al. (1997) contained $\sim 500$ entries. Hence, the number of globular cluster X-ray sources in our M33 catalogue expected from scaling the number of optically known globular clusters is 2-3 and our negative correlation result does not contradict a luminosity function of globular cluster X-ray sources in M 33 similar to that in M33 and the Milky Way.

\subsection{Foreground stars and background objects}

As expected, objects behind and in front of M 33 are found to larger radial distances from the nucleus where optical and radio identifications are easier. All four identified foreground stars (based on position) are located at distances larger than $\sim 15^{\prime}$ from the center of M 33. From the five foreground stars classified from their hardness ratios three are closer to the nucleus for which optical identification will be difficult. Five AGN candidates are suggested from hardness ratios (region III in Fig. 5) and the existence of a radio source or a galaxy (in two cases) as possible counterpart. Two further likely AGN (sources 80 and 98) coincide in position with radio sources which are not confirmed as SNR in the work of Gordon et al. (1999).

Acknowledgements. The ROSAT project is supported by the German Bundesministerium für Bildung, Wissenschaft, Forschung und Technologie (BMBF/DLR) and the Max-PlanckGesellschaft. This research has made use of the SIMBAD data base operated at CDS, Strasbourg, France. Digitized optical images are based on photographic data of the National Geographic Society - Palomar Observatory Sky Survey (NGSPOSS) obtained using the Oschin Telescope on Palomar Mountain. The NGS-POSS was funded by a grant from the National Geographic Society to the California Institute of Technology. The plates were processed into the present compressed digital form with their permission. The Digitized Sky Survey was produced at the Space Telescope Science Institute under US Government grant NAG W-2166.

\section{References}

Boulesteix, J., Courtes, G., Laval, A., Monnet, G., \& Petit, H. 1974, A\&A, 37, 33

Christian, C. A., \& Schommer, R. A. 1982, ApJS, 49, 405

David, L., Harnden, J. F., Kearns, K., \& Zombeck, M. 1996, The ROSAT High Resolution Imager (HRI), USRSDC/SAO Calibration Report, revised

Dubus, G., Charles, P. A., Long, K. S., \& Hakala, P. J. 1997, ApJ, 490, L47

Dubus, G., Charles, P. A., Long, K. S., Hakala, P. J., \& Kuulkers, E. 1999, MNRAS, 302, 731

Duric, N. 2000, in Proceedings 232, WE-Heraeus Seminar, 2225 May 2000, Bad Honnef, Germany, ed. E. M. Berkhuijsen, R. Beck, \& R. A. M. Walterbos (Shaker, Aachen), 2000, 179

Ehle, M., Pietsch, W., \& Beck, R. 1995, A\&A, 295, 289 
Gordon, S. M., Duric, N., Kirshner, R. P., Goss, W. M., \& Viallefond, F. 1999, ApJS, 120, 247

Gordon, S. M., Kirshner, R. P., Long, K. S., et al. 1998, ApJS, 117,89

Haberl, F., Filipović, M. D., Pietsch, W., \& Kahabka, P. 2000, A\&AS, 142, 41

Haberl, F., \& Pietsch, W. 1999, A\&AS, 139, 277

Haberl, F., \& Pietsch, W. 2000, in Proceedings 232. WEHeraeus Seminar, 22-25 May 2000, Bad Honnef, Germany, ed. E. M. Berkhuijsen, R. Beck, \& R. A. M. Walterbos (Shaker, Aachen), 2000, 155

Hodge, P. W., Balsley, J., Wyder, T. K., \& Skelton, B. P. 1999, PASP, 111, 685

Hughes, J. P., Hayashi, I., \& Koyama, K. 1998, ApJ, 505, 732

Kahabka, P., Pietsch, W., \& Hasinger, G. 1994, A\&A, 288, 538

Long, K. S., Charles, P. A., Blair, W. P., \& Gordon, S. M. 1996, ApJ, 466, 750

Long, K. S., Dodorico, S., Charles, P. A., \& Dopita, M. A. 1981, ApJ, 246, L61

Magrini, L., Corradi, R. L. M., Mampaso, A., \& Perinotto, M. 2000, A\&A, 355, 713

Markert, T. H., \& Rallis, A. D. 1983, ApJ, 275, 571

Mochejska, B. J., Kaluzny, J., Krockenberger, M., Sasselov, D. D., \& Stanek, K. Z. 1998, Acta Astron., 48, 455
Monteverde, M. I., Herrero, A., Lennon, D. J., \& Kudritzki, R. 1997, ApJ, 474, L107

Parmar, A. N., Sidoli, L., Oosterbroek, T., et al. 2001, A\&A, 368,420

Pfeffermann, E., Briel, U. G., Hippmann, H., et al. 1987, SPIE, 733,519

Pietsch, W., \& Haberl, F. 2000, in Proceedings 232, WEHeraeus Seminar, 22-25 May 2000, Bad Honnef, Germany, ed. E. M. Berkhuijsen, R. Beck, \& R. A. M. Walterbos (Shaker, Aachen), 2000, 149

Pietsch, W., Vogler, A., Klein, U., \& Zinnecker, H. 2000, A\&A, 360,24

Russell, S. C., \& Dopita, M. A. 1992, ApJ, 384, 508

Sasaki, M., Haberl, F., \& Pietsch, W. 2000, A\&AS, 143, 391

Schulman, E., \& Bregman, J. N. 1995, ApJ, 441, 568

Supper, R., Hasinger, G., Pietsch, W., et al. 1997, A\&A, 317, 328

Trinchieri, G., Fabbiano, G., \& Peres, G. 1988, ApJ, 325, 531

Trümper, J. 1982, Adv. Space Res., 2, 241

van den Bergh, S. 1991, PASP, 103, 609

Zaritsky, D., Elston, R., \& Hill, J. M. 1989, AJ, 97, 97

Zimmermann, H., Becker, W., Belloni, T., et al. 1994, EXSAS User's Guide, MPE Report, 257 